\title{
Numerical Simulation of Fractional Zakharov-Kuznetsov Equation for Description of Temporal Discontinuity Using Projected Differential Transform Method
}

\author{
Dianchen Lu $\mathbb{D},{ }^{1}$ Muhammad Suleman, ${ }^{1,2}$ Jamshaid U1 Rahman $\mathbb{D}^{1},{ }^{1}$ Samad Noeiaghdam, ${ }^{3,4}$ \\ and Ghulam Murtaza $\mathbb{D}^{5}$ \\ ${ }^{1}$ Faculty of Science, Jiangsu University, 212013 Zhenjiang, China \\ ${ }^{2}$ Department of Mathematics, COMSATS University, 44000 Islamabad, Pakistan \\ ${ }^{3}$ Industrial Mathematics Laboratory, Baikal School of BRICS, Irkutsk National Research Technical University, \\ 664074 Irkutsk, Russia \\ ${ }^{4}$ Department of Applied Mathematics and Programming, South Ural State University, \\ Lenin Prospect 76,454080, Chelyabinsk, Russia \\ ${ }^{5}$ School of Science, Department of Mathematics, University of Management and Technology, 54000 Lahore, Pakistan
}

Correspondence should be addressed to Dianchen Lu; dclu@ujs.edu.cn and Jamshaid Ul Rahman; jamshaidrahman@gmail.com

Received 4 March 2021; Accepted 21 June 2021; Published 5 July 2021

Academic Editor: Atila M. Bueno

Copyright (C) 2021 Dianchen Lu et al. This is an open access article distributed under the Creative Commons Attribution License, which permits unrestricted use, distribution, and reproduction in any medium, provided the original work is properly cited.

The core aim of this study is to propose a novel computational procedure, namely, Elzaki transform iterative method to work out two-dimensional nonlinear time-fractional Zakharov-Kuznetsov equation numerically. We execute the suggested iterative procedure on two models and results are presented graphically in the form of surface plot and absolute error is compared with the VIM and HPM to show that the method is more powerful than VIM and HPM and deduce that the offered numerical pattern is more efficient in simulating linear and nonlinear fractional order models.

\section{Introduction}

Applications of fractional calculus are found in various fields such as social science, viscoelasticity, finance, electrochemistry, finance, mathematical physics, signal processing, and physics. In various prominent areas, numerous important models are being found using the fractional derivatives in control, signal theory, mechanics, chemical, acoustics, and fluid and in several other problems which arise in engineering and applied sciences. In real world, we cannot think that any model exits physically without fractional derivatives. There are numerous nonlinear models in this world, and particularly, we say it is not possible to find out the solution analytically of nonlinear fractional models. So, we solve the numerous nonlinear fractional models numerically and computationally. Derivatives and integral order fractional are of important aspects in fractional calculus. It has already been proved by many researchers that fractional order generalizations of integral order models portray the natural phenomenon in extremely proficient manner. The classical derivatives exhibit local nature whereas the Caputo fractional derivatives exhibit nonlocal nature. Such that, making use of the local derivative, we can examine the variation in neighborhood of a point, but applying Caputo fractional derivative, we can examine changes in the interval. Because of this differential characteristic of Caputo fractional derivative, it is appropriate to simulate more physical phenomenon such as vibration, ocean, dynamical system, climate, physics, atmospheric, earthquake, and polymers. Due to vast applications of fractional differential equations, many researchers, such as Senol et al. [1], Sahoo and Ray [2], Das and Saha [3], Porogo et al. [4], Kuo [5], HE [6-8], Liu et al. [9], Shang et al. [10], Wang and Liu [11], Wang et al. [12], $\mathrm{Hu}$ and $\mathrm{He}$ [13], Wu and Liang [14], 
He [15-22], Liu et al. [23], Adamu and Ogenyi [24], El-Dib [25], Filobello-Nino et al. [26], Yildrim [27-29], He [30-34], Ahmad [35], Prakash and Kumar [36], Molliq et al. [37], Kumar et al. [38,39], Aruna and Ravi Kanth [40], DaftardarGejji et al. [41-44], Jafari et al. [45], Bhalekar and DaftardarGejji [46], Munro and Parkes [47], Sakharov and Kuznetsov [48], Kumar et al. [49, 50], Pandey and Mishra [51], Zhang et al. [52], Podlubny [53], Laskin [54], Sun et al. [55], Singh [56], Kumar et al. [57, 58], Yang et al. [59], Suleman et al. [60-64], and Ahmad et al. [65, 66], studied different types of differential equations to understand the physical phenomena. The key issues in physical sciences, such as mathematical physics, are modeled with the help of nonlinear partial differential equations. In the investigation of nonlinear physical phenomenon, fractional order plays a vital role in finding the solution for nonlinear evolution problems.

In this study, we introduced iterative Elzaki transform method to study the numerical solution of two-dimensional nonlinear Zakharov-Kuznetsov equations fractional in time. This iterative Elzaki transform method is a combination of Elzaki transform and projected differential transform method, which provides the solution in a convergent series form.

The Zakharov-Kuznetsov equation is a model describing the isotropic evolution of a nonlinear ion-acoustic wave; many phenomena reveal that the wave travels discontinuously in time, and some properties hidden in a nonlinear wave has to be observed in multiple time scales. For example, for a flow in a tube, the main flow property can be described by the laminar theory, but the vortex near the boundary has to be described in a more small time scale, and a fractional model is needed.

In the current study, nonlinear Zakharov-Kuznetsov equation fractional in time has been studied:

$$
\frac{\partial^{\alpha} \varphi}{\partial t^{\alpha}}+a \frac{\partial \varphi^{p}}{\partial x}+b \frac{\partial^{3} \varphi^{q}}{\partial x^{3}}+c \frac{\partial^{3} \varphi^{r}}{\partial x \partial y^{2}}=0
$$

where $\varphi$ is a function of $x$ and $y, t$ and $\alpha$ are parameter characterizing fractional derivatives $0<\alpha \leq 1, a, b$, and $c$ are real constants, and $p, q$, and $r$ are natural numbers that deal with the behavior of ion-acoustic waves. These waves are barely nonlinear in plasma and consist of cold ions and hot isothermal electrons in the presence of a steady magnetic field. This type of nonlinear equation was in three dimensions and was obtained firstly during the studies of steady magnetized lossless plasma to illustrate weakly ion-acoustic waves. The vital inspiration to work on this idea is established, a computational procedure to investigate nonlinear fractional differential equations, which is reliable and efficient. Since, their application is being found in mathematical modeling of real-world problems.

In this study, we suggest the iterative Elzaki transform method to numerically solve the nonlinear Zakharov-Kuznetsov equation fractional in time. Before this, the Elzaki transform and projected differential transform method has been used by many researchers discretely due to their strong characteristics. We are well familiar that integral transform methods are very helpful in finding the solution of linear and nonlinear fractional, ordinary, and partial differential equations. Previously, lot of articles were found on applications of integral transforms such as Laplace, Fourier, Hankel, and Mellin, but hardly any articles were found on the power series of these integral transforms such as the Elzaki transform. Apparently, this transformation is used not extensively so far by many researchers. The Elzaki transform is very powerful tool to solve linear and nonlinear differential equation, but it has several remarkable advantages over the already used integral transforms. The most useful characteristics of the Elzaki transform is its unity feature, which reduces the computational time to compute nonlinear fractional problems arising in several branches of engineering and applied sciences. The development of the proposed method is based on combination of two strong methodologies and applicable to work with different types of fractional order linear and nonlinear ordinary and partial differential equation. We suggest the proposed method can minimize the work and computational time as compared to the already used methods, while the efficiency is maintained for the approximate results, decreasing the size amount in improvement of the implementation of proposed technique.

\section{Basic Definitions of Fractional Calculus and Elzaki Transform}

(1) On the domain of functions the Elzaki transform can be defined as

$$
B=\left\{g(k)\left|N \cdot k_{1} \cdot k_{2}>0 \cdot\right| g(k) \mid<N e^{\left(|k| k_{j}\right)} \text { if } k \in(-1)^{j} \times(0, \infty)\right\} .
$$

Given formulae can be written in the form

$$
E[g(k)]=\int_{0}^{\infty} g(u k) e^{-k} d k, \quad u \in\left(-k_{1}, k_{2}\right) .
$$

$$
\begin{aligned}
E\left[D_{x}^{n \alpha} u(x, t)\right]= & v^{-n \alpha} E[u(x, t)]-\sum_{k=0}^{m-1} v^{(-n \alpha+k)} u^{k}(0 . t), \\
& n-1<n \alpha \leq n .
\end{aligned}
$$

(2) The Elzaki transform is defined for the Caputo fractional derivative by the following formula:

(3) He's fractional derivative: 


$$
D_{t}^{\alpha} g(t)=D^{\alpha} \frac{d^{n}}{d t^{n}}\left(I^{n} g\right)=\frac{d^{n}}{d t^{n}}\left(I^{n-\alpha} g\right)=\frac{1}{\Gamma(n-\alpha)} \frac{d^{n}}{d t^{n}} \int_{t_{0}}^{t}(v-t)^{n-\alpha-1}\left[g_{0}(v)-g(v)\right] d v
$$

(4) Keeping the first term of $g_{0}(v)$, we give another application of He's fractional derivative in the form

$$
D_{t}^{\alpha} g(t)=\frac{1}{\Gamma(n-\alpha)} \frac{d^{n}}{d t^{n}} \int_{t_{0}}^{t}(v-t)^{n-\alpha-1}\left[g\left(t_{0}\right)-g(v)\right] d v
$$

Note that $g$ can be continuous but possibly not differentiable anywhere.

(5) Variational iteration method (VIM):

$$
D_{t}^{\alpha} g(t)=\frac{1}{\Gamma(n-\alpha)} \frac{d^{n}}{d t^{n}} \int_{t_{0}}^{t}(v-t)^{n-\alpha-1} g(v) d v .
$$

(6) Caputo fractional derivative can be defined in He's fractional derivative as

$$
D_{x}^{\alpha} g(x)=\frac{1}{\Gamma(n-\alpha)} \int_{0}^{x}(x-t)^{n-\alpha-1} \frac{d^{n} g(t)}{d t^{n}} d t
$$

(7) Riemann Liouville derivative can be defined in He's fractional derivative as

$$
D_{x}^{\alpha} g(x)=\frac{1}{\Gamma(n-\alpha)} \frac{d^{n}}{d x^{n}} \int_{0}^{x}(x-t)^{n-\alpha-1} g(t) d t
$$

(8) Local fractional derivative has attracted much attention due to its simple chain rule:

$$
g^{(\alpha)}\left(x_{0}\right)=\left.\frac{d^{\alpha} g(x)}{d x^{\alpha}}\right|_{x=x_{0}}=\lim _{x \longrightarrow x_{0}} \frac{\Delta^{\alpha}\left(g(x)-g\left(x_{0}\right)\right)}{\left(x-x_{0}\right)^{\alpha}}
$$

where

$$
\Delta^{\alpha}\left(g(x)-g\left(x_{0}\right)\right) \cong \Gamma(1+\alpha) \Delta\left(g(x)-g\left(x_{0}\right)\right) .
$$

(9) Fractal derivative:

$$
\frac{D u}{D x^{\alpha}}=\lim _{\Delta x \longrightarrow L_{0}} \frac{(U(A)-U(B))}{k L_{0}^{\alpha}} .
$$

(10) Alternatively,

$$
\frac{D u}{D x^{\alpha}}=\Gamma(1+\alpha) \lim _{\Delta x=x_{A}-x_{B}} \longrightarrow L_{0} \frac{(U(A)-U(B))}{\left(x_{A}-x_{B}\right)^{\alpha}} .
$$

\section{Description of Elzaki Transforms' Iterative Method}

Consider the functional equation $u(z)=g(z)+N u(z)$, where $N$ is the nonlinear operator defined on Banach space A and $g$ is any known function. The main objective is to acquire the solution in convergent series form. Assume $u(z)=\sum_{i=0}^{\alpha} u_{i}(z)$, whereas $N u(z)$ nonlinear operator can be represented as

$$
N[u(z)]=N\left[\sum_{i=0}^{\infty} u_{i}(z)\right]=N\left[u_{0}\right]+\sum_{i=1}^{\infty}\left[N\left(\sum_{j=0}^{i} u_{j}\right)-N\left(\sum_{j=0}^{i-1} u_{j}\right)\right] .
$$

So, we obtain

$u(z)=g(z)+N\left(u_{0}\right)+\sum_{i=1}^{\infty}\left[N\left(\sum_{j=0}^{i} u_{j}\right)-N\left(\sum_{j=0}^{i-1} u_{j}\right)\right]$.

Comparing both sides, we have

$$
\begin{aligned}
u_{0} & =g, \\
u_{1} & =N\left(u_{0}\right), \\
u_{k+1} & =N\left(u_{0}+u_{1}+, \ldots,+u_{k}\right)-N\left(u_{0}+u_{1}+, \ldots,+u_{k-1}\right), \\
k & =1.2 \ldots \ldots
\end{aligned}
$$

Now, consider

$$
D_{t}^{\alpha} u(z, t)+M u(z, t)=g(z, t),
$$

with initial condition,

$$
u(z, 0)=h(z)
$$

where nonlinear operator is denoted by $M$. Now, apply the Elzaki transform on either sides of equation (17), we obtain

$$
E\left[D_{t}^{\alpha} u(z, t)+M u(z, t)\right]=E[g(z, t)] .
$$

Using the differential property of the Elzaki transform, we obtain 


$$
\bar{u}=u(z, 0)+v^{\alpha}[E[g]-E[M u]]=u(z, 0)+v^{\alpha} E[g]-v^{\alpha} E[M u]=G-v^{\alpha} E[M u] .
$$

Using the inverse Elzaki transform, we obtain

$$
u=g(z, t)-E^{-1}\left[v^{\alpha} E[M u]\right],
$$

which is of the type as $u=g+N u$, where $N u=-E^{-1}\left[v^{\alpha} E[M u]\right]$; then,

$$
\begin{aligned}
u_{0} & =G, \\
u_{1} & =-E^{-1}\left[v^{\alpha} E\left[M u_{0}\right]\right], \\
u_{k+1} & =-E^{-1}\left[v^{\alpha} E\left\{M \sum_{j=0}^{i} u_{j}-M \sum_{j=0}^{i-1} u_{j}\right\}\right] .
\end{aligned}
$$

\section{Error Analysis of Proposed Technique}

In this section, the error analysis of proposed technique is presented.

Theorem 1. If there exist a real number $k, 0<k<1$, satisfying, $u_{i+1}(z, v) \leq k u_{i}(z, v)$ for each values of $i$. Furthermore, if we use the truncated series $\sum_{i=0}^{l} u_{i}(z, v)$ to approximate solution of $u(z, t)$. We get the maximum truncated error:

$$
\left\|u(z, v)-\sum_{i=0}^{l} u_{i}(z, v)\right\| \leq \frac{k^{l+1}}{(1-k)}\left\|u_{0}(z, v)\right\| .
$$

Proof. We obtain

$$
\begin{aligned}
& \left\|u(z, v)-\sum_{i=0}^{l} u_{i}(z, v)\right\|=\left\|\sum_{i=l+1}^{\infty} u_{i}(z, v)\right\| \leq \sum_{i=l+1}^{\infty}\left\|u_{i}(z, v)\right\| \leq \sum_{i=l+1}^{\infty} k^{i}\left\|u_{i}(z, v)\right\| \\
& \quad \leq k^{l+1}\left[1+(k)^{1}+(k)^{2}+\ldots\right]\left\|u_{0}(z, v)\right\| \leq \frac{k^{l+1}}{(1-k)}\left\|u_{0}(z, v)\right\|,
\end{aligned}
$$

which completes the proof.

\section{Illustrative Examples}

In this section, we execute the proposed iterative method on fractional Zakharov-Kuznetsov equation.

Example 1. Consider the nonlinear time-fractional Zakharov-Kuznetsov equation:

$$
D_{t}^{\alpha} u+\left(u^{2}\right)_{x}+\frac{1}{8}\left(u^{2}\right)_{x x x}+\frac{1}{8}\left(u^{2}\right)_{x y y}=0,
$$

where $0<\alpha<1$ and given initial condition $u(x, y, 0)=(1 / 3) \sigma\left(e^{(x+y)}-e^{-(x+y)}\right)^{2}$, where $\sigma$ is arbitrary constant.

Exact solution for $\alpha=1$ is

$$
u(x, y, 0)=\frac{1}{3} \sigma\left(e^{(x+y-\sigma t)}-e^{-(x+y-\sigma t)}\right)^{2} .
$$

Applying the Elzaki transform on both sides,

$$
\bar{u}=\frac{1}{3} \sigma\left(e^{(x+y-\sigma t)}-e^{-(x+y-\sigma t)}\right)^{2}+v^{\alpha}\left[E\left[-\left(u^{2}\right)_{x}-\frac{1}{8}\left(u^{2}\right)_{x x x}-\frac{1}{8}\left(u^{2}\right)_{x y y}\right]\right]
$$

Using inverse Elzaki transform, we obtain 


$$
\begin{aligned}
u(x, y, t)= & \frac{1}{3} \sigma\left(e^{(x+y-\sigma t)}-e^{-(x+y-\sigma t)}\right)^{2}+E^{-1}\left[v^{\alpha} E\left[-\left(u^{2}\right)_{x}-\frac{1}{8}\left(u^{2}\right)_{x x x}-\frac{1}{8}\left(u^{2}\right)_{x y y}\right]\right] \\
u_{0}(x, y, t)= & \frac{1}{3} \sigma\left(e^{(x+y-\sigma t)}-e^{-(x+y-\sigma t)}\right)^{2}, \\
u_{1}(x, y, t)= & -\frac{2}{3} \frac{\sigma^{2} t^{\alpha}}{\Gamma(1+\alpha)}\left(e^{(x+y)}-e^{-(x+y)}\right)\left(e^{3(x+y)}-e^{-3(x+y)}+3\left(e^{(x+y)}-e^{-(x+y)}\right)\right), \\
u_{2}(x, y, t)= & \frac{12800}{27} \frac{\Gamma(1+2 \alpha) \sigma^{4} t^{3 \alpha}}{\Gamma(1+\alpha)^{2} \Gamma(1+3 \alpha)}\left(e^{6(x+y)}-e^{-6(x+y)}\right)-\frac{2560}{81} \frac{\Gamma(1+2 \alpha) \sigma^{4} t^{3 \alpha}}{\Gamma(1+\alpha)^{2} \Gamma(1+3 \alpha)}\left(e^{2(x+y)}-e^{-2(x+y)}\right) \\
& -\frac{5120}{81} \frac{\Gamma(1+2 \alpha) \sigma^{4} t^{3 \alpha}}{\Gamma(1+\alpha)^{2} \Gamma(1+3 \alpha)}\left(e^{4(x+y)}-e^{-4(x+y)}\right)-\frac{54400}{81} \frac{\Gamma(1+2 \alpha) \sigma^{4} t^{3 \alpha}}{\Gamma(1+\alpha)^{2} \Gamma(1+3 \alpha)}\left(e^{8(x+y)}-e^{-8(x+y)}\right) \\
& +\frac{800}{9} \frac{\sigma^{3} t^{2 \alpha}}{\Gamma(1+2 \alpha)}\left(e^{6(x+y)}-e^{-6(x+y)}\right)+\frac{416}{27} \frac{\sigma^{3} t^{2 \alpha}}{\Gamma(1+2 \alpha)}\left(e^{2(x+y)}-e^{-2(x+y)}\right)-\frac{2240}{27} \frac{\sigma^{3} t^{2 \alpha}}{\Gamma(1+2 \alpha)}\left(e^{4(x+y)}-e^{-4(x+y)}\right) \\
& +\frac{16}{9} \frac{\sigma^{2} t^{\alpha}}{\Gamma(1+\alpha)}\left(e^{2(x+y)}-e^{-2(x+y)}\right)-\frac{20}{9} \frac{\sigma^{2} t^{\alpha}}{\Gamma(1+\alpha)}\left(e^{4(x+y)}-e^{-4(x+y)}\right) \\
& +\frac{2}{3} \frac{\sigma^{2} t^{\alpha}}{\Gamma(1+\alpha)}\left(e^{(x+y)}-e^{-(x+y)}\right)\left(e^{3(x+y)}-e^{-3(x+y)}+3\left(e^{(x+y)}-e^{-(x+y)}\right)\right) \\
& +\frac{14}{9} \frac{\sigma^{2} t^{\alpha}}{\Gamma(1+\alpha)}\left(e^{3(x+y)}-e^{-3(x+y)}+3\left(e^{(x+y)}-e^{-(x+y)}\right)\right) .
\end{aligned}
$$

Similarly, we can find the higher order terms of $u(x, y, t)$ using the proposed method. Finally, we get the approximate solution $u(x, y, t)$ denoted by equation (28) is $(x, y, 0)=\sum_{i=0}^{\infty} u_{i}$.

For $\alpha=1, t=0.5$, and $\sigma=0.001$, illustrate absolute error, numerical solution, and exact solution in Figures 1-3. On comparison, we found that numerical solution obtained by the Elzaki transform iterative method is identical to exact solution shown in Figures 1-3. We used third order approximation to estimate the efficiency, absolute error, and approximate solution obtained by the Elzaki transform iterative method. Comparison between approximate and exact solution to obtain absolute error for specific value of $x$ and $t$ with other techniques is presented in the tabular form (Table 1).
Example 2. Consider $(3,3,3)$ time-fractional Z-K equation:

$$
D_{t}^{\alpha} u+\left(u^{3}\right)_{x}+2\left(u^{3}\right)_{x x x}+2\left(u^{3}\right)_{x y y}=0, \quad 0<\alpha<1 .
$$

Initial condition:

$$
u(x, y, 0)=\frac{3}{4} \sigma\left(e^{(x+y / 6)}-e^{-(x+y / 6)}\right) .
$$

For $\alpha=1$, the exact solution of equation (29) is

$$
u(x, y, t)=\frac{3}{4} \sigma\left(e^{(x+y-\sigma t / 6)}-e^{-(x+y-\sigma t / 6)}\right) .
$$

Using the differential properties of Elzaki transformation on both sides of equation (29), we obtain

$$
\bar{u}=\frac{3}{4} \sigma\left(e^{(x+y-\sigma t / 6)}-e^{-(x+y-\sigma t / 6)}\right)+v^{\alpha} E\left[-\left(u^{3}\right)_{x}-2\left(u^{3}\right)_{x x x}-2\left(u^{3}\right)_{x y y}\right] .
$$

Applying the inverse Elzaki transform, we obtain

$$
u(x, y, t)=\frac{3}{4} \sigma\left(e^{(x+y-\sigma t / 6)}-e^{-(x+y-\sigma t / 6)}\right)+E^{-1}\left[v^{\alpha} E\left[-\left(u^{3}\right)_{x}-2\left(u^{3}\right)_{x x x}-2\left(u^{3}\right)_{x y y}\right]\right] .
$$




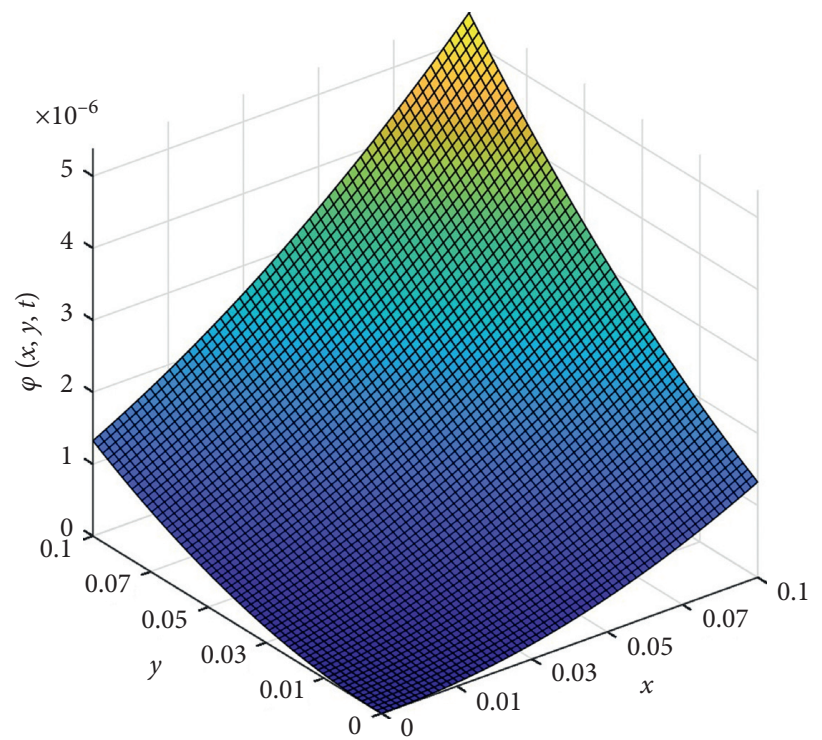

FIgURE 1: Represents exact solution of Example 1 at $\alpha=1$.

Using the Elzaki iterative method, the successive terms are as follows:

$$
\begin{aligned}
& u_{0}(x, y, t)=\frac{3}{4} \sigma\left(e^{(x+y / 6)}-e^{-(x+y / 6)}\right) \\
& u_{1}(x, y, t)=-\frac{3 \sigma^{2} t^{\alpha}}{64 \Gamma(1+\alpha)}\left(e^{(x+y / 2)}-e^{-(x+y / 2)}+3\left(e^{(x+y / 6)}-e^{-(x+y / 6)}\right)\right) \\
& u_{2}(x, y, t)=\frac{23031 \sigma^{9} t^{4 \alpha} \Gamma(1+3 \alpha)}{65536 \Gamma(1+\alpha)^{3} \Gamma(1+4 \alpha)}\left(e^{(x+y / 2)}-e^{-(x+y / 2)}\right) \\
& +\frac{295245 \sigma^{9} t^{4 \alpha} \Gamma(1+3 \alpha)}{262144 \Gamma(1+\alpha)^{3} \Gamma(1+4 \alpha)}\left(e^{(3 x+3 y / 2)}-e^{-(3 x+3 y / 2)}\right)-\frac{11475 \sigma^{9} t^{4 \alpha} \Gamma(1+3 \alpha)}{65536 \Gamma(1+\alpha)^{3} \Gamma(1+4 \alpha)}\left(e^{(5 x+5 y / 6)}-e^{-(5 x+5 y / 6)}\right) \\
& -\frac{5325 \sigma^{9} t^{4 \alpha} \Gamma(1+3 \alpha)}{131072 \Gamma(1+\alpha)^{3} \Gamma(1+4 \alpha)}\left(e^{(x+y / 6)}-e^{-(x+y / 6)}\right)-\frac{246645 \sigma^{9} t^{4 \alpha} \Gamma(1+3 \alpha)}{262144 \Gamma(1+\alpha)^{3} \Gamma(1+4 \alpha)}\left(e^{(7 x+7 y / 6)}-e^{-(7 x+7 y / 6)}\right) \\
& -\frac{2025 \sigma^{7} t^{3 \alpha} \Gamma(1+2 \alpha)}{16384 \Gamma(1+\alpha)^{2} \Gamma(1+3 \alpha)}\left(e^{(x+y / 2)}-e^{-(x+y / 2)}\right)-\frac{4155 \sigma^{7} t^{3 \alpha} \Gamma(1+2 \alpha)}{65536 \Gamma(1+\alpha)^{2} \Gamma(1+3 \alpha)}\left(e^{(x+y / 6)}-e^{-(x+y / 6)}\right) \\
& +\frac{43605 \sigma^{7} t^{3 \alpha} \Gamma(1+2 \alpha)}{16384 \Gamma(1+\alpha)^{2} \Gamma(1+3 \alpha)}\left(e^{(5 x+5 y / 6)}-e^{-(5 x+5 y / 6)}\right)-\frac{49329 \sigma^{7} t^{3 \alpha} \Gamma(1+2 \alpha)}{16384 \Gamma(1+\alpha)^{2} \Gamma(1+3 \alpha)}\left(e^{(7 x+7 y / 6)}-e^{-(7 x+7 y / 6)}\right) \\
& +\frac{105 \sigma^{5} t^{2 \alpha}}{512 \Gamma(1+2 \alpha)}\left(e^{(x+y / 6)}-e^{-(x+y / 6)}\right)+\frac{2295 \sigma^{5} t^{2 \alpha}}{1024 \Gamma(1+2 \alpha)}\left(e^{(5 x+5 y / 6)}-e^{-(5 x+5 y / 6)}\right) \\
& -\frac{1863 \sigma^{5} t^{2 \alpha}}{1024 \Gamma(1+2 \alpha)}\left(e^{(x+y / 2)}-e^{-(x+y / 2)}\right)+\frac{51 \sigma^{3} t^{\alpha}}{2048 \Gamma(1+\alpha)}\left(e^{(x+y / 2)}-e^{-(x+y / 2)}+3\left(e^{(x+y / 6)}-e^{-(x+y / 6)}\right)\right) \\
& -\frac{27 \sigma^{3} t^{\alpha}}{64 \Gamma(1+\alpha)}\left(e^{(x+y / 2)}-e^{-(x+y / 2)}\right)+\frac{15 \sigma^{3} t^{\alpha}}{64 \Gamma(1+\alpha)}\left(e^{(x+y / 6)}-e^{-(x+y / 6)}\right) \\
& +\frac{27 \sigma^{3} t^{\alpha}}{64 \Gamma(1+\alpha)}\left(e^{(x+y / 3)}-e^{-(x+y / 3)}-2\right)\left(e^{(x+y / 6)}-e^{-(x+y / 6)}\right) .
\end{aligned}
$$




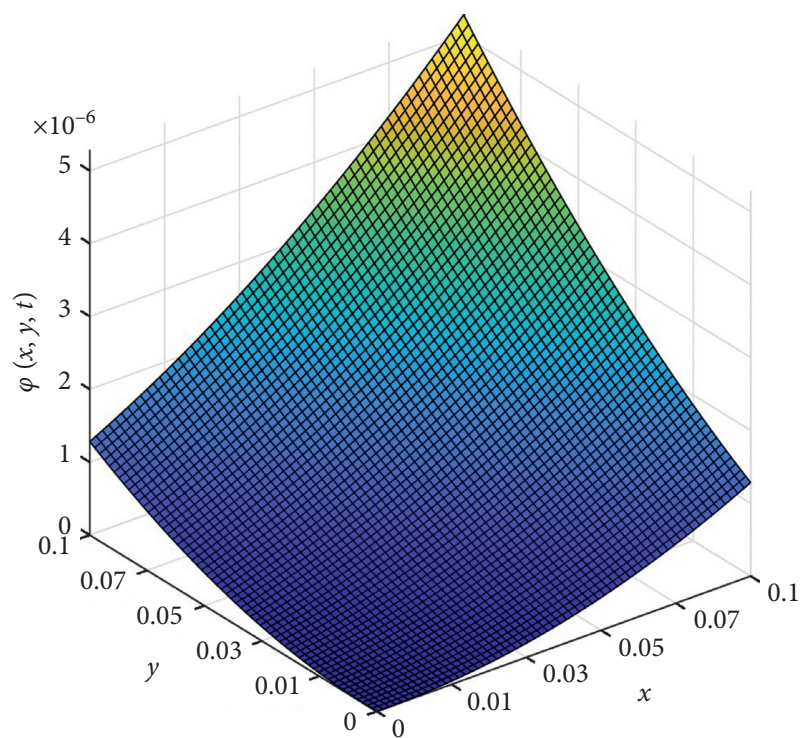

FIgURE 2: Represents numerical solution of Example 1 at $\alpha=1$.

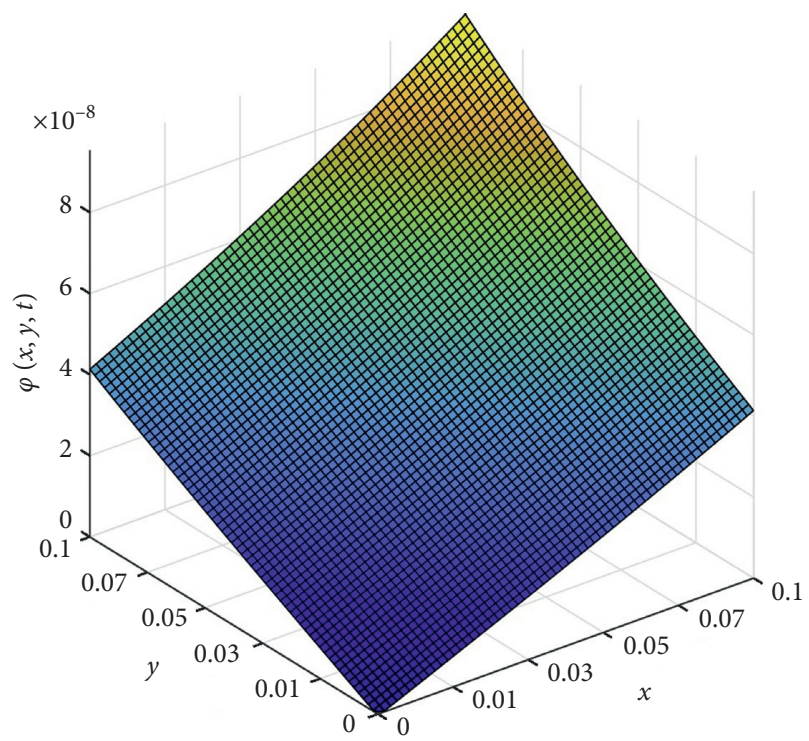

Figure 3: Represents absolute error of Example 1 at $\alpha=1$.

TABLE 1: Comparison among different methods for absolute error when $\alpha=1$ and $\sigma=0.001$.

\begin{tabular}{cccccc}
\hline$x$ & $t$ & ETIM & VIM & HPTM & NISTM \\
\hline \multirow{4}{*}{0.0} & 0.0 & $2.00 \times 10^{-8}$ & $2.00 \times 10^{-8}$ & $2.00 \times 10^{-8}$ & $2.00 \times 10^{-8}$ \\
& 0.01 & $1.50 \times 10^{-7}$ & $3.99 \times 10^{-6}$ & $1.50 \times 10^{-7}$ & $1.40 \times 10^{-7}$ \\
& 0.03 & $3.06 \times 10^{-7}$ & $8.01 \times 10^{-6}$ & $3.06 \times 10^{-7}$ & $3.10 \times 10^{-7}$ \\
& 0.05 & $5.55 \times 10^{-7}$ & $1.59 \times 10^{-5}$ & $5.55 \times 10^{-7}$ & $5.55 \times 10^{-7}$ \\
& 0.07 & $7.66 \times 10^{-7}$ & $2.24 \times 10^{-5}$ & $7.80 \times 10^{-7}$ & $7.80 \times 10^{-7}$ \\
& 0.10 & $8.12 \times 10^{-7}$ & $2.01 \times 10^{-5}$ & $8.11 \times 10^{-7}$ & $8.12 \times 10^{-7}$ \\
\hline \multirow{4}{*}{0.01} & 0.0 & $1.40 \times 10^{-7}$ & $3.95 \times 10^{-6}$ & $1.40 \times 10^{-7}$ & $1.40 \times 10^{-7}$ \\
& 0.01 & $3.25 \times 10^{-7}$ & $8.01 \times 10^{-6}$ & $3.01 \times 10^{-7}$ & $3.01 \times 10^{-7}$ \\
& 0.03 & $5.53 \times 10^{-7}$ & $9.19 \times 10^{-6}$ & $4.65 \times 10^{-7}$ & $4.65 \times 10^{-7}$ \\
& 0.05 & $7.32 \times 10^{-7}$ & $2.05 \times 10^{-5}$ & $6.35 \times 10^{-7}$ & $8.11 \times 10^{-7}$ \\
& 0.07 & $8.11 \times 10^{-7}$ & $2.11 \times 10^{-5}$ & $8.11 \times 10^{-7}$ & $8.11 \times 10^{-7}$ \\
& 0.10 & $9.98 \times 10^{-7}$ & $2.41 \times 10^{-7}$ & $9.99 \times 10^{-7}$ & $9.98 \times 10^{-7}$ \\
\hline & & & & &
\end{tabular}

TABle 1: Continued.

\begin{tabular}{|c|c|c|c|c|c|}
\hline$x$ & $t$ & ETIM & VIM & HPTM & NISTM \\
\hline \multirow{6}{*}{0.03} & 0.0 & $3.01 \times 10^{-7}$ & $7.90 \times 10^{-7}$ & $3.01 \times 10^{-7}$ & $3.01 \times 10^{-7}$ \\
\hline & 0.01 & $4.75 \times 10^{-7}$ & $1.49 \times 10^{-6}$ & $4.66 \times 10^{-7}$ & $4.66 \times 10^{-7}$ \\
\hline & 0.03 & $5.63 \times 10^{-7}$ & $1.89 \times 10^{-6}$ & $6.63 \times 10^{-7}$ & $6.63 \times 10^{-7}$ \\
\hline & 0.05 & $8.91 \times 10^{-7}$ & $2.11 \times 10^{-5}$ & $8.11 \times 10^{-7}$ & $7.55 \times 10^{-7}$ \\
\hline & 0.07 & $9.40 \times 10^{-6}$ & $2.23 \times 10^{-5}$ & $9.97 \times 10^{-7}$ & $8.11 \times 10^{-6}$ \\
\hline & 0.10 & $1.2 \times 10^{-6}$ & $2.94 \times 10^{-5}$ & $2.41 \times 10^{-6}$ & $1.2 \times 10^{-6}$ \\
\hline \multirow{6}{*}{0.05} & 0.0 & $4.66 \times 10^{-7}$ & $1.19 \times 10^{-6}$ & $4.66 \times 10^{-7}$ & $4.66 \times 10^{-7}$ \\
\hline & 0.01 & $5.67 \times$ & $1.34 \times 10^{-6}$ & $6.35 \times$ & 6.35 \\
\hline & 0.03 & $7.22 \times 10$ & $1.98 \times 10^{-5}$ & $8.12 \times 10^{-7}$ & $\times 10^{-7}$ \\
\hline & 0.05 & $8.98 \times 10^{-7}$ & $2.00 \times 10^{-5}$ & $9.98 \times 10^{-7}$ & $9.98 \times 10^{-7}$ \\
\hline & 0.07 & $1.01 \times 10^{-7}$ & $2.41 \times 10^{-5}$ & $1.20 \times 10^{-7}$ & $1.20 \times 10^{-7}$ \\
\hline & 0.10 & $1.2 \times 10^{-7}$ & $3.27 \times 10^{-5}$ & $1.41 \times 10^{-7}$ & $1.41 \times 10^{-7}$ \\
\hline \multirow{6}{*}{0.07} & & & $1.55 \times 10^{-6}$ & $6.35 \times 10^{-7}$ & $6.35 \times 10^{-7}$ \\
\hline & 0.01 & & $1.89 \times 10^{-6}$ & $7.12 \times$ & $<10^{-7}$ \\
\hline & 0.03 & $7.22 \times 10^{-7}$ & $2.11 \times 10^{-6}$ & $8.34 \times 10^{-7}$ & $7.22 \times 10^{-7}$ \\
\hline & 0.05 & $8.98 \times 10^{-7}$ & $8.98 \times 10^{-5}$ & $9.98 \times 10^{-7}$ & $8.98 \times 10^{-7}$ \\
\hline & 0.07 & & $3.52 \times 10^{-5}$ & $1.20 \times 10^{-6}$ & $\times 10^{-7}$ \\
\hline & 0.10 & $.2 \times 10^{-7}$ & $2.48 \times 10^{-5}$ & $1.56 \times 10^{-6}$ & $10^{-7}$ \\
\hline \multirow{6}{*}{0.10} & & & $2.00 \times 10^{-6}$ & $8.12 \times 10^{-7}$ & $\times 10^{-7}$ \\
\hline & 0.01 & $8.98 \times 10^{-7}$ & $2.21 \times 10^{-6}$ & $9.98 \times 10^{-7}$ & $8.98 \times 10^{-7}$ \\
\hline & 0.03 & $7.22 \times 10^{-6}$ & $2.64 \times 10^{-5}$ & $522 \times 10^{-6}$ & $7.22 \times 10^{-6}$ \\
\hline & 0.05 & & $3.27 \times 10^{-5}$ & $3.35 \times 10^{-6}$ & $6.35 \times 10^{-6}$ \\
\hline & 0.07 & & $3.77 \times 10^{-5}$ & $2.66 \times 10^{-6}$ & $4.66 \times 10^{-6}$ \\
\hline & 0.10 & $1.63 \times 10^{-6}$ & $4.11 \times 10^{-6}$ & $1.88 \times 10^{-6}$ & $1.63 \times 10^{-6}$ \\
\hline
\end{tabular}

Similarly, we can find the higher order terms of $u(x, y, t)$ using the proposed method. Finally, we get the approximate solution $u(x, y, t)$ is given by $(x, y, 0)=\sum_{i=0}^{\infty} u_{i}$.

For $\alpha=1, t=0.5$, and $\sigma=0.001$, illustrate absolute error, numerical solution, and exact solution in Figures 4-6. On comparison, we found that numerical solution obtained by the Elzaki transform iterative method is identical to the exact solution shown in Figures 4-6. We used third-order approximation to estimate the efficiency, absolute error, and approximate solution obtained by the Elzaki transform iterative method. Comparison between approximate and exact solution to obtain absolute error for different value of $x$ and $t$ with other methods is presented in Table 2 .

\section{Results and Discussion}

On comparison of numerical and exact solution in Figures 1 and 2 at $\alpha=1, t=0.5$, and $\sigma=0.001$ for nonlinear $(2,2,2)$ Zakharov-Kuznetsov equation fractional in time and $u$ strictly increases with the increase in $x$. Comparison shows exceptional concurrency between numerical and exact solutions. In Figure 3, we demonstrate if $x$ increases at $y=1, t=1$, and $\sigma=0.001$, respectively, and at $\alpha=0.75,1$. $u$ strictly increases and $\alpha=0.25,0.5$. From Figure 4 , it is now obvious that the solution depends on fractional derivative of time. In Figure 5, we evaluate exact and numerical solution for standard case of time-fractional Zakharov-Kuznetsov. When $\alpha=1, t=0.5, \sigma=0.001$, then the nonlinear time $(3,3,3)$ time-fractional Zakharov-Kuznetsov $\mathrm{u}$ increases strictly with the $\mathrm{x}$ increases. Figure 6 demonstrates $\alpha$ at $y=1, t=1$, and $\sigma=0.001$, numerous values of fractional order, respectively. It is pragmatic from Figure 6 
TABLE 2: Absolute error for Example 1 between two consecutive approximate terms at different values of $\alpha$ for unknown exact solution.

\begin{tabular}{cccccc}
\hline$x$ & $t$ & $\alpha=0.5\left|\varphi_{2}-\varphi_{1}\right|$ & $\alpha=0.5\left|\varphi_{3}-\varphi_{2}\right|$ & $\alpha=0.75\left|\varphi_{2}-\varphi_{1}\right|$ & $\alpha=0.5\left|\varphi_{3}-\varphi_{2}\right|$ \\
\hline 0.0 & 0.0 & $4.27 \times 10^{-8}$ & $6.57 \times 10^{-11}$ & $3.21 \times 10^{-8}$ & $2.11 \times 10^{-8}$ \\
0.01 & 0.01 & $4.12 \times 10^{-8}$ & $8.98 \times 10^{-11}$ & $4.98 \times 10^{-8}$ & $3.98 \times 10^{-11}$ \\
0.03 & 0.03 & $4.98 \times 10^{-8}$ & $7.22 \times 10^{-10}$ & $5.35 \times 10^{-8}$ & $6.88 \times 10^{-10}$ \\
0.05 & 0.05 & $6.35 \times 10^{-8}$ & $1.35 \times 10^{-9}$ & $8.11 \times 10^{-7}$ & $1.65 \times 10^{-9}$ \\
0.07 & 0.07 & $8.11 \times 10^{-7}$ & $4.66 \times 10^{-9}$ & $9.63 \times 10^{-7}$ & $3.33 \times 10^{-9}$ \\
0.10 & 0.10 & $1.63 \times 10^{-7}$ & $7.63 \times 10^{-9}$ & & $5.98 \times 10^{-9}$ \\
\hline
\end{tabular}

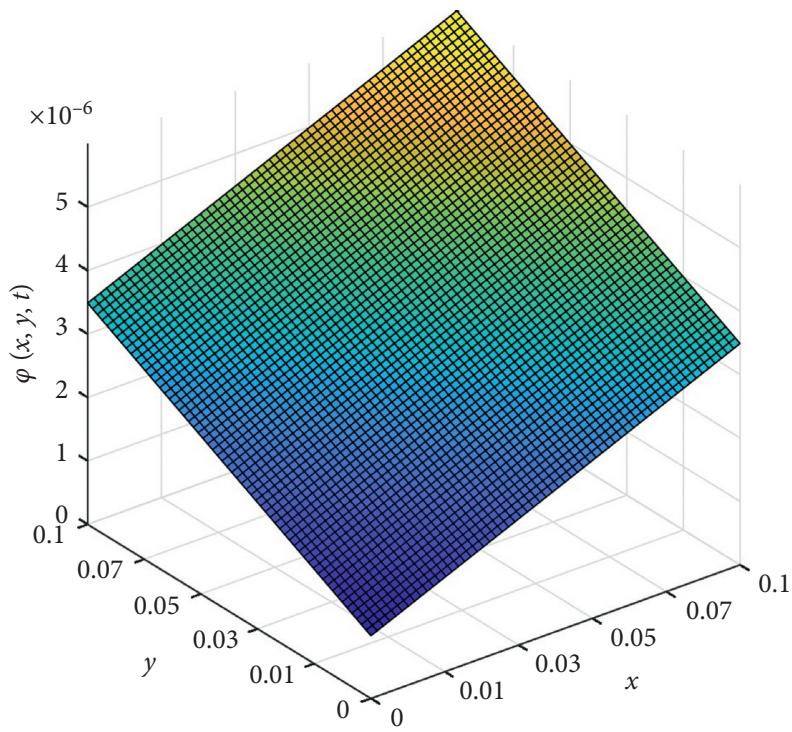

Figure 4: Represents exact solution of Example 2 at $\alpha=1$.

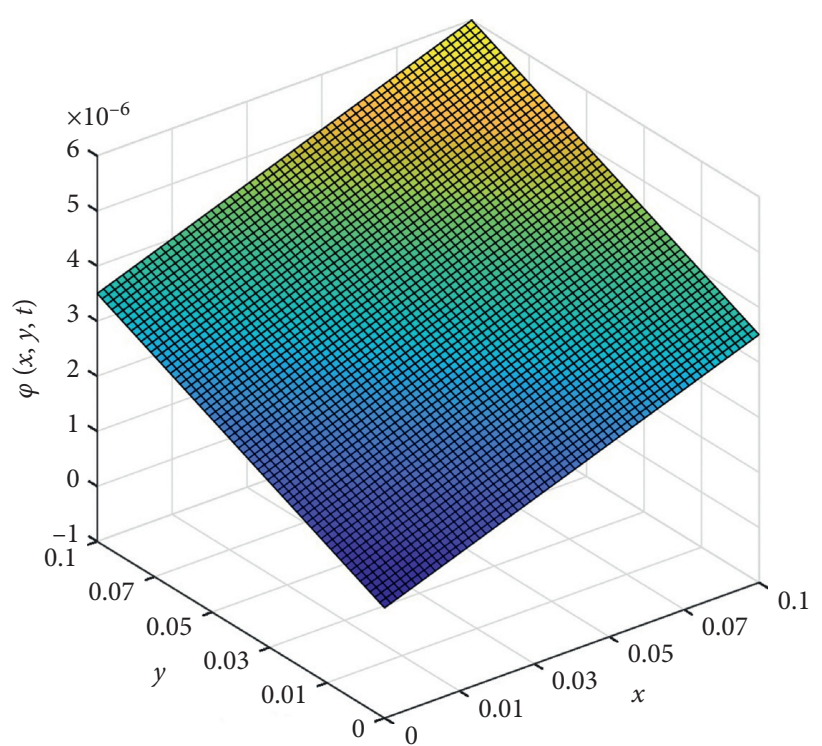

Figure 5: Represents numerical solution of Example 2 at $\alpha=1$.

that $u$ increases strictly with the increase in $x$. Table 2 demonstrates that the absolute error can be neglected between the consecutive iterations because it approaches 0 , and
Table 3 represents the absolute error for Example 1 between two consecutive approximate terms at different values of $\alpha$ for unknown exact solution. 


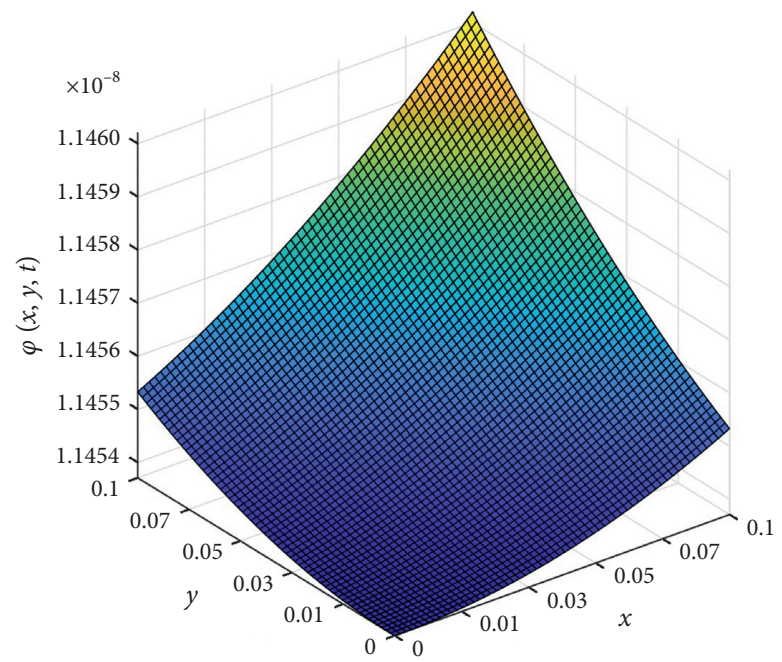

FIGURE 6: Represents absolute error of Example 2 at $\alpha=1$.

TABLE 3: Absolute error for Example 1 between two consecutive approximate terms at different values of $\alpha$ for unknown exact solution.

\begin{tabular}{cccccc}
\hline$x$ & $t$ & $\alpha=0.5\left|\varphi_{2}-\varphi_{1}\right|$ & $\alpha=0.5\left|\varphi_{3}-\varphi_{2}\right|$ & $\alpha=0.75\left|\varphi_{2}-\varphi_{1}\right|$ & $\alpha=0.5\left|\varphi_{3}-\varphi_{2}\right|$ \\
\hline 0.0 & 0.0 & $1.98 \times 10^{-10}$ & $7.91 \times 10^{-21}$ & $1.91 \times 10^{-10}$ & $4.27 \times 10^{-21}$ \\
0.01 & 0.01 & $1.98 \times 10^{-10}$ & $3.22 \times 10^{-20}$ & $1.91 \times 10^{-10}$ & $7.88 \times 10^{-20}$ \\
0.03 & 0.03 & $1.99 \times 10^{-10}$ & $5.99 \times 10^{-18}$ & $1.92 \times 10^{-10}$ & $3.76 \times 10^{-10}$ \\
0.05 & 0.05 & $1.99 \times 10^{-10}$ & $5.55 \times 10^{-18}$ & $1.93 \times 10^{-10}$ & $5.84 \times 10^{-18}$ \\
0.07 & 0.07 & $2.00 \times 10^{-10}$ & $7.55 \times 10^{-17}$ & $1.93 \times 10^{-10}$ & $3.88 \times 10^{-17}$ \\
0.10 & 0.10 & $2.00 \times 10^{-10}$ & $2.23 \times 10^{-17}$ & $7.66 \times 10^{-17}$ \\
\hline
\end{tabular}

Fractional order $\alpha$ number of iteration increases. This shows the application of proposed method for the fractional order.

\section{Conclusion}

In this study, we used ETNM to obtain the solution of nonlinear Zakharov-Kuznetsov equation fractional in time. We attain the solution in the convergent series form. The basic characterization of ETNM to find solution of nonlinear models is without using restrictive assumption, which is the improvement over other methods. Numerical comparison shows that this method is more powerful than VIM and HPM. These methods also work well for the unknown integer order. Consequently, we deduce that the presented numerical scheme is efficient and powerful in solving linear and nonlinear fractional order models.

\section{Data Availability}

The data used to support the findings of this study are included within the article.

\section{Disclosure}

The authors declare that this manuscript is original, has not been published before, and is not currently being considered for publication elsewhere.

\section{Conflicts of Interest}

The authors declare that they have no conflicts of interest.

\section{Authors' Contributions}

All authors contributed to this research. Material preparation, methodology, data collection, formal analysis, and software were performed by Muhammad Suleman, Jamshaid Ul Rahman, and Dianchen Lu. Initially, the manuscript was prepared by Muhammad Suleman and modified as per the suggestions by the other authors. Final version was reviewed and edited by Samad Noeiaghdam, Jamshaid Ul Rahman, and Ghulam Murtaza. All authors approved the final manuscript.

\section{Acknowledgments}

This work was supported by National Natural Science Foundation, China, and Faculty of Science, Jiangsu University, Zhenjiang, China.

\section{References}

[1] M. Senol, M. Alquran, and H. D. Kasmaei, "On the comparsion of perturbation-iteration algorithm and residual power series method to solve fractional Zakharov-Kuznetsov equation," Results in Physics, vol. 9, pp. 321-327, 2018. 
[2] S. Sahoo and S. S. Ray, "Improved sub-equation method for (3+1) dimensional generalized fractional KdV-Zakharov-Kuznetsov equations," Computers \& Mathematics with Applications, vol. 70, no. 2, pp. 158-166, 2015.

[3] A. Das and A. Saha, "Dynamical survey of the dual power Zakharov-Kuznetsov-Burgers equation with external periodic perturbation," Computers \& Mathematics with Applications, vol. 76, no. 5, pp. 1174-1183, 2018.

[4] O. P. Porogo, B. Muatjeteja, and A. R. Adem, "Variational approach and exact solutions for a generalized coupled Zakharov-Kuznetsov system," Computers \& Mathematics with Applications, vol. 73, no. 5, pp. 864-872, 2017.

[5] C. K. Kuo, "The new exact solitary and multi-soliton solutions for the $(2+1)$ dimensional Zakhraov- Kuznetsov equation," Computers \& Mathematics with Applications, vol. 75, no. 8, pp. 2851-2857, 2018.

[6] J.-H. He, "A tutorial review on fractal spacetime and fractional calculus," International Journal of Theoretical Physics, vol. 53, no. 11, pp. 3698-3718, 2014.

[7] J.-H. He, "Fractal calculus and its geometrical explanation," Results in Physics, vol. 10, no. S1, pp. 272-276, 2018.

[8] J.-H. He, "A new fractal derivation," Thermal Science, vol. 15, no. 1, pp. 145-147, 2011.

[9] H. Y. Liu, Z. M. Liu, and F. K. Ko, "A fractional model for heat transfer in Mongolian yurt," Thermal Science, vol. 21, no. 4, pp. 1861-1866, 2017.

[10] X. Shang, J. Wang, and X. Yang, "Fractal analysis for heat extraction in geothermal system," Thermal Science, vol. 21, no. 1, pp. 25-31, 2017.

[11] K. L. Wang and S. Y. Liu, "He's fractional derivative and its application for fractional Fornberg-Whitham equation," Thermal Science, vol. 21, no. 5, pp. 2049-2055, 2017.

[12] Y. Wang, Y. Zhang, and W. Rui, "Shallow water waves in porous medium for coast protection," Thermal Science, vol. 21, no. 1, pp. 145-151, 2017.

[13] Y. Hu and J.-H. He, "On fractal space-time and fractional calculus," Thermal Science, vol. 20, no. 3, pp. 773-777, 2016.

[14] X. E. Wu and Y. S. Liang, "Relationship between fractal dimensions and fractional calculus," Nonlinear Science Letters A, vol. 8, pp. 77-89, 2017.

[15] J. H. He, S. K. Elagan, and Z. B. Li, "Geometrical explanation of the fractional complex transform and derivative chain rule for fractional calculus," Physics Letters A, vol. 376, no. 4, pp. 257-259, 2012.

[16] J. H. He and Z. B. Li, "Converting fractional differential equations into partial differential equations," Thermal Science, vol. 16, no. 2, pp. 331-334, 2016.

[17] J.-H. He, "Homotopy perturbation technique," Computer Methods in Applied Mechanics and Engineering, vol. 178, no. 3-4, pp. 257-262, 1999.

[18] J.-H. He, "A coupling method of a homotopy technique and a perturbation technique for non-linear problems," International Journal of Non-linear Mechanics, vol. 35, no. 1, pp. 37-43, 2000.

[19] J.-H. He, "Some asymptotic methods for strongly nonlinear equations," International Journal of Modern Physics B, vol. 20, no. 10, pp. 1141-1199, 2006.

[20] J. H. He, "Homotopy perturbation method with an auxiliary term," Abstract and Applied Analysis, vol. 2012, Article ID 857612, 2012.

[21] J. H. He, "Homotopy perturbation method with two expanding parameters," Indian Journal of Physics, vol. 88, no. 2, pp. 193-196, 2014.
[22] Y. Wu and J.-H. He, "Homotopy perturbation method for nonlinear oscillators with coordinate-dependent mass," Results in Physics, vol. 10, pp. 270-271, 2018.

[23] Z.-J. Liu, M. Adamu, E. Suleiman, and J.-H. He, "Hybridization of homotopy perturbation method and Laplace transformation for the partial differential equations," Thermal Science, vol. 21, no. 4, pp. 1843-1846, 2017.

[24] M. Y. Adamu and P. Ogenyi, "Parameterized homotopy perturbation method," Nonlinear Science Letters A, vol. 8, no. 2, pp. 240-243, 2017.

[25] Y. O. El-Dib, "Multiple scales homotopy perturbation method for nonlinear oscillators," Nonlinear Science Letters A, vol. 8, pp. 352-364, 2017.

[26] U. Filobello-Nino, H. Vazquez-Leal, K. Boubaker et al., "Nonlinearities distribution homotopy perturbation method to find solution for Troesch's problem," Nonlinear Science Letters A, vol. 8, no. 3, pp. 279-291, 2018.

[27] A. Yildirim, "An algorithm for solving the fractional nonlinear Schrödinger equation by means of the homotopy perturbation method," International Journal of Nonlinear Sciences and Numerical Simulation, vol. 10, no. 4, pp. 445-450, 2009.

[28] A. Yildinm, "Analytical approach to Fokker-Planck equation with space- and time-fractional derivatives by means of the homotopy perturbation method," Journal of King Saud University - Science, vol. 22, no. 4, pp. 257-264, 2010.

[29] A. Yildirim and Y. Gulkanat, "Analytical approach to fractional Zakharov-Kuznetsov equations by He's homotopy perturbation method," Communications in Theoretical Physics, vol. 53, no. 6, pp. 1005-1010, 2010.

[30] J. H. He, "Variational iteration method- a kind of non-linear analytical technique: some examples," International Journal of Non-Linear Mechanics, vol. 34, no. 4, pp. 699-708, 1999.

[31] J. H. He, "Variational Iteration method- some recent results and new interpretations," Journal of Computational and Applied Mathematics, vol. 207, no. 1, pp. 3-17, 2007.

[32] J. H. He and X. H. Wu, "Variational iteration method: new development and applications," Computers \& Mathematics with Applications, vol. 54, no. 7-8, pp. 881-894, 2007.

[33] J. He, "Variational iteration method for delay differential equations," Communications in Nonlinear Science and Numerical Simulation, vol. 2, no. 4, pp. 235-236, 1997.

[34] J. H. He, "An approximate solution technique depending on an artificial parameter: a special example," Communications in Nonlinear Science and Numerical Simulation, vol. 3, no. 2, pp. 92-97, 1998.

[35] H. Ahmad, "Auxiliary parameter in the variational iteration algorithm-II and its optimal determination," Nonlinear Science Letters A, vol. 9, no. 1, pp. 62-72, 2018.

[36] A. Prakash and M. Kumar, "He's variational iteration method for the solution of nonlinear Newell-Whitehead-Segel equation," Journal of Applied Analysis \& Computation, vol. 6, no. 3, pp. 738-748, 2016.

[37] R. Y. Molliq, M. S. M. Noorani, I. Hashim, and R. R. Ahmad, "Approximate solutions of fractional Zakharov-Kuznetsov equations by VIM," Journal of Computational and Applied Mathematics, vol. 233, no. 2, pp. 103-108, 2009.

[38] S. Kumar, H. Kocak, and A. Yildirim, "A fractional model of gas dynamics equations and its analytical approximate solution using Laplace transform," Zeitschrift für Naturforschung A, vol. 67, no. 6-7, pp. 389-396, 2012.

[39] S. Kumar, A. Yildirim, Y. Khan, and L. Wei, "A fractional model of the diffusion equation and its analytical solution using Laplace transform," Iranian Journal of Science and 
Technology, Transaction B: Engineering, vol. 19, no. 4, pp. 1117-1123, 2012.

[40] K. Aruna and A. S. V. Ravi Kanth, "Approximate solutions of nonlinear fractional Schrodinger equation via differential transform method and modified differential transform method," National Academy Science Letters, vol. 36, no. 2, pp. 201-221, 2013.

[41] V. Daftardar-Gejji and H. Jafari, "An iterative method for solving nonlinear functional equations," Journal of Mathematical Analysis and Applications, vol. 316, no. 2, pp. 753-763, 2006.

[42] V. Daftardar-Gejji and S. Bhalekar, "Solving fractional boundary value problems with Dirichlet boundary conditions using a new iterative method," Computers \& Mathematics with Applications, vol. 59, no. 5, pp. 1801-1809, 2010.

[43] S. Bhalekar and V. Daftardar-Gejji, "New iterative method: application to partial differential equations," Applied Mathematics and Computation, vol. 203, no. 2, pp. 778-783, 2008.

[44] V. Daftardar-Gejji and S. Bhalekar, "Solving fractional diffusion-wave equations using the new iterative method," Fractional Calculus and Applied Analysis, vol. 11, no. 2, pp. 193-202, 2008.

[45] H. Jafari, S. Seifi, A. Alipoor, and M. Zabihi, "An iterative method for solving linear and nonlinear fractional diffusionwave equation," Journal of Nonlinear Fractional and Phenomenal Science in Engineering, vol. 11, no. 2, 2007.

[46] S. Bhalekar and V. Daftardar-Gejji, "Solving evolution equations using a new iterative method," Numerical Methods for Partial Differential Equations, vol. 26, no. 4, pp. 906-916, 2010.

[47] S. Munro and E. J. Parkes, "Stability of solitary-wave solutions to a modified Zakharov-Kuznetsov equation," Journal of Plasma Physics, vol. 64, no. 4, pp. 411-426, 2000.

[48] V. E. Zakharov and E. A. Kuznetsov, "Three-dimensional solutions," Soviet Physics Uspekhi, vol. 39, pp. 285-286, 1974.

[49] D. Kumar, J. Singh, and S. Kumar, "Numerical computation of nonlinear fractional Zakharov-Kuznetsov equation arising in ion-acoustic waves," Journal of the Egyptian Mathematical Society, vol. 22, no. 3, pp. 373-378, 2014.

[50] D. Kumar, J. Singh, and D. Baleanu, "A hybrid computational approach for Klein-Gordon equations on Cantor sets," Nonlinear Dynamics, vol. 87, no. 1, pp. 511-517, 2016.

[51] R. K. Pandey and H. K. Mishra, "Numerical simulation for solution of space-time fractional telegraphs equations with local fractional derivatives via HAFSTM," New Astronomy, vol. 57, pp. 82-93, 2017.

[52] Y. Zhang, H. Sun, H. H. Stowell, M. Zayernouri, and S. E. Hansen, "A review of applications of fractional calculus in Earth system dynamics," Chaos, Solitons \& Fractals, vol. 102, pp. 29-46, 2017.

[53] I. Podlubny, Fractional Differential Equations, Academic Press, New York, NY, USA, 1999.

[54] N. Laskin, "Time fractional quantum mechanics," Chaos, Solitons \& Fractals, vol. 102, pp. 16-28, 2017.

[55] H. Sun, Z. Li, Y. Zhang, and W. Chen, "Fractional and fractal derivative models for transient anomalous diffusion: model comparison," Chaos, Solitons \& Fractals, vol. 102, pp. 346353, 2017.

[56] H. Singh, "Approximate solution of fractional vibration equation using Jacobi polynomials," Applied Mathematics and Computation, vol. 317, pp. 85-100, 2018.

[57] D. Kumar, J. Singh, and S. Kumar, "A fractional model of Navier-Stokes equation arising in unsteady flow of a viscous fluid," Journal of the Association of Arab Universities for Basic and Applied Sciences, vol. 17, no. 1, pp. 14-19, 2015.

[58] D. Kumar, J. Singh, and D. Baleanu, "Numerical computation of a fractional model of differential-difference equation," Journal of Computational and Nonlinear Dynamics, vol. 11, no. 6, 2016.

[59] S. Yang, H. Fu, and B. Yu, "Fractal analysis of flow resistance in tree like branching networks with roughened micro channels," Fractals, vol. 25, no. 1, Article ID 1750008, 2017.

[60] M. Suleman, D. Lu, J. He, U. Farooq, H. S. Ye, and J. Ul Rahman, "Numerical investigation of fractional HIV model using Elzaki projected differential transform method," Fractals, vol. 26, no. 5, Article ID 1850062, 2018.

[61] M. Suleman, D. Lu, J. He, U. Farooq, S. Noeiaghdam, and F. Ali Chandio, "Elzaki projected differential transform method for fractional order system of linear and nonlinear fractional partial differential equation," Fractals, vol. 26, no. 3, Article ID 1850041, 2018.

[62] J. Ul Rahman, M. Suleman, D. C. Lu, J.-H. He, and M. Ramzan, "He-Elzaki method for spatial diffusion of biological population," Fractals, vol. 27, no. 5, Article ID 1950069, 2019.

[63] D. Lu, M. Suleman, M. Ramzan, and J. Ul Rahman, "Numerical solutions of coupled nonlinear fractional $\mathrm{KdV}$ equations using He's fractional calculus," International Journal of Modern Physics B, vol. 35, no. 2, Article ID 2150023, 2020.

[64] J. Rahman, U. Khan, S. Ahmad et al., "Numerical simulation of Darcy-forchheimer 3D unsteady nanofluid flow comprising carbon nanotubes with cattaneo-christov heat flux and velocity and thermal slip conditions," Processes, vol. 7, no. 10, p. 687, 2019.

[65] M. Ahmad, M. A. Imran, M. Aleem, and I. Khan, "A comparative study and analysis of natural convection flow of MHD non-Newtonian fluid in the presence of heat source and first-order chemical reaction," Journal of Thermal Analysis and Calorimetry, vol. 137, no. 5, pp. 1783-1796, 2019.

[66] M. A. Imran, I. Khan, M. Ahmad, N. A. Shah, and M. Nazar, "Heat and mass transport of differential type fluid with noninteger order time-fractional Caputo derivatives," Journal of Molecular Liquids, vol. 229, pp. 67-75, 2017. 